\title{
CONSCIENCIA DEL VALOR DISTINTIVO DE LOS FONEMAS CONSONÁNTICOS EN POSICIÓN DE NEUTRALIZACIÓN EN EL ESPAÑOL DE CHILE*
}

\section{Edgardo Cifuentes Becerra**}

\section{Resumen}

En algunos autores y escuelas fonológicas en particular, se encuentra presente la idea de que en posición de neutralización la mayoría de los hablantes dejan de ser conscientes de la diferencia fonémica que les resulta clara en posiciones de pertinencia. En este trabajo se estudia la consciencia de los hablantes sobre la identidad de los fonemas involucrados en una neutralización, a través de un test de percepción. Se encuentra que existe una relación entre la ausencia de consciencia y 3 factores que debilitan la claridad de una oposición fonológica: conformar una oposición bilateral, ocurrir en distensión silábica y la asimilación fonética a un segmento circundante. La conjunción de estos factores también permite dar cuenta de la diferencia entre neutralizaciones generalizadas en la lengua castellana y otras que ocurren sólo en algunos dialectos.

Palabras clave: Neutralización fonémica, consciencia, oposición fonológica.

\section{AWARENESS OF THE DISTINCTIVE VALUE OF THE CONSONANT PHONEMES IN NEUTRALIZATION POSITION IN THE SPANISH OF CHILE}

\begin{abstract}
The idea that, in neutralization position, most of speakers are unaware of the phonemic differences which is clear in a pertinent position is present in some authors and phonological schools. In this research, the awareness of speakers about the phonemes identity involved in neutralization was studied, using a perception test. Our findings showed that there is a relationship between awareness absence and 3 factors that hinders the clarity on phonological contrasts, i.e.: conforming a bilateral contrast, being in a syllabic distention and a phonetic assimilation to a surrounding segment. Combination of these factors also shed light on the difference among generalized neutralizations in Spanish and others which only occur in some dialects.
\end{abstract}

Keywords: Phonemic neutralization, awareness, phonologic opposition.

Recibido: 01-07-2015

Aceptado: 21-01-2016

* Este artículo se inscribe en la investigación de tesis doctoral titulada "Neutralización fonémica socialmente condicionada como mecanismo de innovación léxica", financiada por una beca CONICYT de doctorado nacional.

** Chileno. Doctor (c) en Lingüística, Universidad de Concepción, Concepción, Chile. ecifuentes@ udec.cl 


\section{Introducción}

El objetivo de este trabajo es observar el comportamiento de los hablantes respecto a la neutralización, específicamente a propósito de la constatación de creaciones léxicas que involucran neutralizaciones fonémicas. En un trabajo anterior (Cifuentes, 2015) hemos dado a conocer el fenómeno del uso de las formas dostor, úrtimo, güena, juerte y humirde (pronunciadas o escritas de esta forma no estándar) para referirse a significados distintos, aunque relacionados, de sus equivalentes estándares doctor, último, buena, fuerte y humilde. En este trabajo se ha presentado numerosa evidencia que indica que los hablantes del español de Chile usan las formas no estándares cuando quieren referirse a las acepciones: 'falso médico', 'vulgar', 'atractiva', 'de olor o sabor intenso' y 'pobre', respectivamente; además, se ha demostrado que los hablantes hacen una diferencia entre estas formas y sus equivalentes estándares, para las cuales prefieren los significados que el diccionario registra como los principales. El resultado de este fenómeno es que cada palabra se escinde en dos formas que se oponen en el habla informal y en la escritura no estándar. De este modo, estos casos se constituyen en una especie de dobletes etimológicos emergentes.

El proceso mediante el cual se gestan estos dobletes emergentes muestra numerosas similitudes con la descripción de la formación de algunos dobletes etimológicos (los formados por "semicultismos") que ofrecen autores como Wright (1982) y Anderson (1991): pares de formas fonológicas que en un principio alternan en variación libre, progresivamente van especializando su ámbito de uso hasta llegar a diferenciar su significado. En los casos que hemos descrito, una neutralización fonémica de uso restringido a ciertos registros o grupos sociales genera dos formas fonológicas distintas, que alternan en variación libre en la mayor parte de los casos, excepto en un número reducido de palabras donde las formas comienzan a diferenciar su significado según se pronuncien o escriban en la forma estándar o neutralizada. El resultado es que las neutralizaciones recobran su valor distintivo en esas palabras.

Visto lo anterior, parece necesario observar con atención las neutralizaciones para determinar qué características de éstas facilitan el cambio semántico, fonológico y la consiguiente bifurcación léxica, necesarios para que surja un doblete. Trubetzkoy afirma que las 
oposiciones constantes son fácilmente distinguibles para cualquier hablante, mientras que las neutralizables tienen una estrecha semejanza, de modo que para la mayoría de los hablantes no será posible diferenciar qué sonido se produjo en una posición de neutralización (1987 [1939], p. 70). Evidentemente, esto sería un impedimento para retomar el valor distintivo de los fonemas, por lo que deben existir diferencias a este respecto en el comportamiento de las neutralizaciones: en algunas de ellas los hablantes sí deben ser capaces de distinguir qué sonido se produjo para poder atribuir valor distintivo a estos sonidos. Esta posibilidad de distinguir qué sonido se produce en una neutralización demuestra que a veces los hablantes tienen conciencia del valor distintivo de los fonemas, aún en una posición en que se neutralizan. La conciencia del valor distintivo de los fonemas que participan en una neutralización es el objeto de estudio específico de este trabajo.

\section{Neutralización}

La neutralización fonémica es un concepto enmarcado en la fonología funcionalista y relacionado estrechamente con la escuela de Praga. El funcionalismo, como teoría lingüística, se considera continuador de las ideas de Saussure respecto a la naturaleza sistémica de las lenguas y del valor relacional de las unidades que integran el sistema. Por esto, concibe el nivel fonológico como un subsistema de la lengua, compuesto de unidades cuyo valor está determinado por su oposición a las demás unidades del sistema: los fonemas. Éstos son unidades de función, pues representan oposiciones funcionales entre sonidos, lo cual se interpreta en la fonología praguense como la posibilidad de establecer distinciones de significado.

La teoría debía hacerse cargo de un caso de excepción a esta definición del fonema. En algunas posiciones de la sílaba, los fonemas pierden su valor distintivo, por lo que ya no sirven para conformar oposiciones. Este hecho se evidencia en la imposibilidad de conmutación, que es el procedimiento probatorio utilizado en el funcionalismo para identificar los fonemas.

Algunas escuelas funcionalistas resolvieron este problema planteando la existencia de distintos sistemas fonológicos en una misma lengua (multisistematismo), cada uno de los cuales funcionaría en una determinada posición silábica (Rodríguez, 1990, pasa revista a ésta y 
otras soluciones propuestas desde el funcionalismo para este problema). Sin embargo, la propuesta que más prosperó fue la de la neutralización y el archifonema. Esta tesis afirma que en las posiciones en que el valor distintivo de los fonemas se suspende (se neutraliza), aparece una unidad segmental no especificada, cuyos rasgos pertinentes son los que poseen en común los fonemas que participan de esta suspensión de la distintividad. A esta unidad se la llamó archifonema (Trubetzkoy, 1987 [1939]).

\subsection{Diferencias de comportamiento en distintas neutralizaciones}

La neutralización es un fenómeno que no se manifiesta de forma homogénea. La mayoría de los autores que la tratan con algún detalle, dan cuenta de diferencias importantes entre las distintas neutralizaciones, incluso las que se dan en una misma lengua. La siguiente lista reúne algunos de los criterios que pueden establecer tales diferencias y los tipos que resultan de la aplicación de estos criterios:

1) Realización fonética: a) en posición de neutralización, se realiza un fono que no es alófono de ninguno de los fonemas cuyo contraste se neutraliza; b) el archifonema se realiza con un alófono de alguno de los fonemas neutralizados, seleccionado por influencia de los segmentos circundantes; c) la realización fonética en posición de neutralización corresponde al miembro no marcado de la oposición (en el caso de las oposiciones privativas) o del miembro extremo (en las oposiciones graduales); d) ambos miembros de la oposición pueden representar al archifonema (Trubetzkoy, 1987 [1939], p. 71-75; Alarcos, 1965, p. 5051).

2) Condicionamiento: a) la neutralización se produce en contacto con determinados fonemas; b) la neutralización se produce en determinadas posiciones dentro de la palabra (Trubetzkoy, 1987 [1939], p. 209-221; Alarcos, 1965, p. 97-99).

3) Representación fonémica: a) la unidad segmental que aparece en posición de neutralización se puede representar como un archifonema, es decir, una unidad que no corresponde a ninguno de los fonemas cuya oposición se neutraliza; b) la unidad segmental corresponde a uno de los fonemas, pero en posición de neutralización éste asume la 
forma de un alófono propio del otro fonema (Burquest, 2009, p. 6773).

4) Conciencia del hablante: a) en algunos casos, la diferencia entre los fonemas involucrados es irrelevante para los hablantes (en esa posición silábica), por lo que la neutralización ocurre siempre, en todos los estilos de habla; b) en otros casos, los hablantes son conscientes de una alteración en los fonemas que componen la palabra cuando ocurre neutralización, por lo que en habla cuidada corrigen esta ambigüedad; de este modo, la neutralización ocurre sólo en el habla informal (Obediente, 1998, p. 298-230).

\subsection{Neutralización de consonantes en español de Chile}

Los autores que describen el sistema fonológico del español (en sus distintas variedades) asumiendo la hipótesis fonémica, dan cuenta de la existencia de las siguientes neutralizaciones de oposiciones consonánticas: $/ \mathrm{p}-\mathrm{b} /, / \mathrm{k}-\mathrm{g} /, / \mathrm{r}-\mathrm{r} /, / \mathrm{m}-\mathrm{n}-\mathrm{n} /$, todas en coda silábica (Alarcos, 1965; Stockwell y Bowen, 1965; Quilis, 1999); la neutralización de $/ \mathrm{r}-\mathrm{r} /$ en inicio de palabra es controversial, ya que Alarcos (1965) y Stockwell y Bowen (1965) interpretan la ausencia de [r] en inicial de palabra como neutralización, mientras que Quilis (1999) la interpreta como distribución defectiva de / $/$; la neutralización de /1 - r - r/ o / $1-\mathrm{R} /$ ocurre en algunos dialectos del español (Alarcos, 1965; Stockwell y Bowen, 1965). Obediente (1998), quien describe las neutralizaciones que ocurren en el español de Venezuela, incluyendo en su inventario todas las antes mencionadas y agregando la neutralización de los archifonemas / B - D - G/ en un solo archifonema de segundo grado, considera la posibilidad de agregar a esta serie el fonema /s / (en atención a ejemplos de pronunciación como [ah.'moh.fe.ra], por /a.'mos.fe.ra/), pero finalmente rechaza esta alternativa por considerar este fenómeno como distinto en esencia a la neutralización, por motivos que más adelante mencionaremos. Morales Pettorino (1976) describe las neutralizaciones del español de Chile, considerando los mismos casos del inventario de Obediente, con algunos añadidos: a) agrega la / s / a la serie de los archifonemas oclusivos (aceptando la interpretación que Obediente desecha); b) da cuenta de dos neutralizaciones hasta ahora no mencionadas: $/ \mathrm{b}-\mathrm{g} / \mathrm{y} / \mathrm{f}-\mathrm{x} /$, en ataque complejo, frente a [u] (como en [a.'yuxe.lo] o [a.'xue.ra]). 
Veiga (1984; 1993) propone un análisis completamente distinto. Afirma que, en posición de distensión silábica, se neutralizan todas las consonantes interruptas (oclusivas, nasales y $/ \mathfrak{y} /$ ) en un mismo archifonema que se opone a un archifonema de rasgo líquido y otro fricativo. Argumenta que la presencia y distintividad de las oclusivas en coda, sólo ocurre en el dialecto estándar por influjo latinizante de la escritura, y que en la lengua natural -aún en los dialectos cultos- lo que predomina es la elisión o sustitución por otros sonidos. Este radical desacuerdo está sostenido en base a un muy meticuloso análisis de rasgos binarios, pero con poca referencia a datos empíricos. Por ejemplo, se afirma que la elisión o sustitución de las oclusivas es lo más habitual en el habla informal, sin ofrecerse cuantificaciones de ningún tipo al respecto. En contraposición a esta afirmación, los datos de Cepeda (1994) -que corresponden al habla semiespontánea de entrevista sociolingüísticamuestran que las consonantes oclusivas en coda silábica se realizan mayoritariamente con fonos oclusivos. Para este trabajo no seguiremos este análisis, por el motivo recién expuesto y porque la consciencia de la identidad fonémica en posición de neutralización de oposiciones más precisas (como las que señalan los otros autores aquí referidos) supone la consciencia de las oposiciones más generales que señala Veiga.

\subsection{Caracterización de las neutralizaciones}

Las neutralizaciones que se dan en el español de Chile pueden describirse según los criterios expuestos en 2.1., del siguiente modo:

1) Respecto al primer criterio, la realización fonética, es necesario hacer una precisión que permita distinguirlo del criterio 2, ya que ambos tienen en común un caso: la influencia de los segmentos circundantes. Es difícil distinguir entre la influencia de los segmentos circundantes en la realización fonética de un archifonema (criterio 1) y la influencia de los segmentos circundantes en el hecho mismo de que ocurra una neutralización (criterio 2). Sin embargo, no es el mismo fenómeno. En el primer caso, la influencia de los segmentos circundantes se reduce a definir cuál será la realización fonética de una neutralización que ya existe por otros motivos; en el segundo, se afirma que la neutralización misma se produce a causa del entorno lingüístico.

En el caso del español, la influencia de los segmentos circundantes se encuentra en la neutralización de $/ \mathrm{m}-\mathrm{n}-\mathrm{n} /$, de $/ \mathrm{b}-\mathrm{g} / \mathrm{y}$ de 
/ $\mathrm{f}-\mathrm{x} /$. Es difícil saber si en estas neutralizaciones hay influencia de los segmentos circundantes solamente en la determinación de la realización fonética del segmento o si la coarticulación de alguna forma causa la neutralización. Hay al menos una diferencia importante: /b, g/ y / $\mathrm{f}, \mathrm{x} /$ se neutralizan solamente ante [u], en posición silábica de ataque; las nasales, en cambio, se neutralizan en coda silábica (como la mayoría de las neutralizaciones en español), y no siempre al asimilarse a una consonante, pues también puede darse la neutralización ante pausa. Por lo tanto, podríamos afirmar que en las nasales la condición de neutralización (criterio 2) es la coda silábica, y que la influencia articulatoria de la consonante siguiente repercute en la realización fonética de la unidad neutralizada (criterio 1); mientras tanto, en $/ \mathrm{b}-\mathrm{g} / \mathrm{y} / \mathrm{f}-\mathrm{x} /$, la condición de neutralización (criterio 2) es la influencia articulatoria del segmento semivocálico.

En este punto, los autores no siempre han establecido la diferencia. Por ejemplo, Alarcos afirma que las nasales se neutralizan condicionadas por dos factores: posición silábica ante pausa y asimilación ante consonante (1965: § 58); Veiga rectifica este análisis constatando que la verdadera condición, en ambos contextos de neutralización, es la posición silábica (1995, p. 353-355). Salvo este caso, no se mencionan otras neutralizaciones en que el contexto determine la realización fonética del archifonema.

2) Respecto al condicionamiento de las neutralizaciones (criterio 2), el condicionamiento "interno" del que habla Trubetzkoy (es decir, cuando la neutralización se circunscribe a un lugar de la sílaba o la palabra) parece ser la regla general en español. La excepción la constituye la neutralización de $/ \mathrm{b}-\mathrm{g} / \mathrm{y}$ de $/ \mathrm{f}-\mathrm{x} /$, como ya se mencionó, que está condicionada por el contacto con [u्र].

3) El criterio 3, respecto de la adscripción fonémica de la unidad neutralizada, tiene sentido en la fonología heredera de cierta escuela del distribucionalismo estadounidense. Pike (1961) propone que las modificaciones morfológicas son una evidencia para adscribir un fono a una unidad fonémica. Con este criterio, si bien en la palabra [ba.'lon] el sonido final podría corresponder a un archifonema nasal /N/ (por no existir otras nasales en esta posición), la adición del morfema de plural -es y la conservación del sonido [n] en [ba.'lo. nes] permiten concluir que la unidad subyacente a [n] es el fonema 
/n/. Siguiendo un análisis equivalente, Burquest (2009) concluye que hay neutralizaciones en que la unidad fonológica corresponde a un archifonema y otras en que se puede adscribir a uno de los fonemas que se neutralizan. Un ejemplo del español, equivalente al que analiza Burquest, es el siguiente: la vibrante múltiple [r] en final de palabra -como sucede, por ejemplo, en la pronunciación enfática [mar] - debe interpretarse como realización del fonema $/ \mathrm{f} /$, debido a que realiza como [r] en ['ma.res]; sin embargo, por principio de univocidad (es decir, que un fono es alófono solamente de un fonema en el sistema), no puede interpretarse este hecho como una simple alofonía y debe postularse una neutralización en que la unidad subyacente es un fonema determinado y no un archifonema.

Al aplicar este principio de análisis, resulta que: 1) las nasales no se neutralizan en final de palabra; 2) las vibrantes se neutralizan al final de palabra, aunque sin ocurrir un archifonema; 3) las vibrantes no se neutralizan en inicio de palabra (lo que se demuestra al añadir un prefijo a una palabra que inicia con vibrante, como en [rom.'per] / [i.rom.'pi'.ßle]); 4) las oclusivas dentales se neutralizan al final de palabra, aunque no en un archifonema, pues se puede identificar la

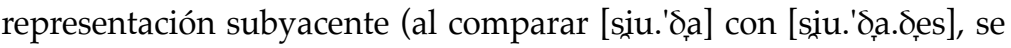
puede adscribir al fonema $/ \mathrm{d} /$ ); 5 ) en el caso de $/ 1-\mathrm{r}-\mathrm{r} /$, cuando se neutralicen su adscripción fonémica dependerá de la producción del hablante: si el plural de delantal [de.la.'ar] se pronuncia [de.la.'a. les], [r] debe adscribirse a $/ 1 /$; 6 ) en los demás casos, en posición de neutralización se postula una unidad archifonémica, ya que, al ir en posición intermedia de palabra, no hay información morfofonológica que permita suponer un fonema $\mathrm{u}$ otro.

4) Finalmente, respecto del criterio 4 sobre la conciencia del hablante (es decir, si realmente los fonemas que se neutralizan son confundidos por los hablantes), la mayoría de los autores no establecen diferencias. Obediente afirma que en la neutralización de los archifonemas / B - D - G/ y el fonema /s/, los hablantes no perciben el conjunto como una sola unidad, pues únicamente sucede en habla coloquial, mientras que en habla enfática los hablantes hacen la distinción (1998, p. 300); la restricción de la neutralización al registro coloquial sería para él una señal de que los hablantes están conscientes del contraste entre estas unidades fonológicas (lo cual el autor interpreta como señal de que no ocurre una verdadera neutralización). 
Otros autores no tienen en cuenta el criterio de consciencia del valor distintivo ni de restricción al registro coloquial, pero sí reparan sobre la adscripción dialectal, estrechamente relacionada con la variación de registro, pues los usos propios de dialectos estigmatizados suelen relegarse al estilo informal. Se describen como "dialectales" y propias del habla "vulgar" las neutralizaciones de / B - D - G - s / (Morales Pettorino, 1976), de /1, R/ (Alarcos, 1965; Morales Pettorino, 1976; Granados, 2001), de /b-g/ y de /f - x/ (Morales Pettorino, 1976). A partir de aquí se puede deducir que las demás neutralizaciones son transversales a todos los registros y dialectos, y que los hablantes no tienen consciencia -en posición de neutralización- del contraste de los fonemas neutralizados.

\subsection{Consciencia del valor distintivo de los fonemas neutralizados}

Los dos últimos criterios de distinción entre neutralizaciones -la identidad fonológica de la unidad neutralizada y la consciencia del hablante de la misma- sugieren una noción psicológica del fonema: se pretende identificar la unidad fonológica que intuye el hablante en su gramática mental inconsciente. Esta idea no es extraña a la fonología, pero está lejos de ser ampliamente aceptada. Las primeras aproximaciones al concepto de fonema -propuestas por algunos precursores como Courtenay, Sapir y otros-, lo describieron primordialmente como unidad psicológica(Gutiérrez, 1983, p.76-77). Posteriormente, el funcionalismo de la escuela de Praga definió el fonema como unidad de función distintiva, es decir, útil para establecer oposiciones léxicas. La naturaleza del fonema se definió, entonces, como unidad de relación entre los componentes del sistema fonológico de una lengua, y en función del significado. Buena parte de la fonología de herencia funcionalista adhiere a esta definición del fonema, y por lo tanto, no considera aspectos psicológicos al analizar la fonología de una lengua.

En la fonología estructuralista norteamericana se distinguen dos perspectivas respecto de la naturaleza psicológica de fonema. Por una parte, un pensamiento de influencia conductista y continuador del distribucionalismo propuesto por Bloomfield se avoca a la tarea de determinar el inventario fonémico de una lengua con la única información de la distribución de los sonidos, sin alusión al significado o la intuición (psicología) de los hablantes. Por otra parte, Sapir -cuyas 
ideas surgieron en estrecha relación con el trabajo de descripción de lenguas sin escritura y poco conocidas- daba especial atención a las intuiciones del hablante nativo en la descripción de la fonología de una lengua, y resaltaba cómo los datos de distribución complementaria y de variaciones morfofonológicas confirmaban estas intuiciones (Sapir, 1972). Las ideas de Sapir se desarrollaron a la par del concepto funcionalista praguense y del concepto distribucionalista del fonema, y su influencia de puede rastrear en algunas ideas de Swadesh y, más claramente, en la fonémica de Pike y sus seguidores (Gutiérrez, 1983).

En esta línea de pensamiento, Pike definió el fonema del siguiente modo: "Los sonidos de una lengua son organizados automática e inconscientemente por el hablante nativo en unidades estructurales que podemos llamar FONEMAS" (Pike, 1961, p. 57). La traducción es nuestra). Burquest, un representante actual de esta segunda línea de pensamiento, afirma que "la fonología se ocupa primordialmente de la organización mental (pero subconsciente) de los sonidos de una lengua en particular" (2009, p. 2), y también que la representación fonémica "debe corresponder de manera más cercana a las intuiciones del hablante nativo sobre igualdad y diferenciación" (p. 41). En atención a estas premisas es que Burquest, como vimos en el apartado anterior, no atribuye de inmediato una representación archifonémica a una neutralización: debe comprobar antes que para los hablantes realmente no hay una diferencia en aquella posición. En este modelo, si el fonema es una unidad mental intuitiva para los hablantes, el archifonema también debería serlo.

A pesar de las diferencias de perspectiva, esta noción de fonema no es del todo ajena al funcionalismo praguense. Cuando Trubetzkoy describe la neutralización, hace referencia a algunos aspectos que podrían considerarse psicológicos: afirma que en la neutralización los hablantes en general no se percatan de las diferencias de fonemas (1939, § III.2.C). Esta indicación, si bien puede no entenderse necesariamente como una caracterización del aspecto psicológico del fonema, al menos sugiere la existencia de una correlación que aporta datos al estudio del sistema. El concepto de neutralización de Veiga, en cambio, no tiene componentes psicológicos; se apega estrictamente a la descripción sistémica, y como resultado postula unidades archifonémicas que parecen poco intuitivas, como las que hemos mencionado en 2.3. 
El acercamiento de la fonología funcionalista a ciertas nociones psicológicas del fonema no es casual. La fonología praguense tiende a concentrarse en el sistema fonológico de modo más bien aislado (aunque la oposición fonémica supone una relación con el significado), pero el principio funcionalista es que los sonidos no son una estructura cerrada, sino que están al servicio de la comunicación entre hablantes. Si bien es cierto, los fonemas establecen relaciones paradigmáticas entre ellos, también es cierto que estas relaciones están determinadas por el uso que los hablantes hacen de los sonidos con fines comunicativos. De aquí podría seguirse la necesidad de que las distinciones pertinentes para el sistema sean también relevantes para los hablantes.

De este modo, las ideas del fonema como unidad psicológica no están en contradicción abierta con el principio funcionalista. De hecho, Burquest pretende conjugar ambas ideas, pues se refiere a su manual de fonología como un "enfoque funcional", que define como la voluntad de explicar los fenómenos fonológicos "desde el punto de vista de la función que desempeñan en el contexto comunicativo de la lengua" (2009, p. xi). Un ejemplo de aplicación de este principio se puede observar en la explicación de la frecuente simetría en los sistemas fonológicos de las lenguas; Burquest afirma que los sistemas fonológicos de las lenguas tienden a ser simétricos porque "Las diferencias entre los sonidos son más fáciles de percibir si se distribuyen los sonidos de una manera uniforme en el espacio fonológico y si se utilizan los extremos de dicho espacio [...]. Esto aligera la carga impuesta sobre el usuario de la lengua en lo que respecta al desciframiento del mensaje" (Burquest, 2009, p. 9-10).

En esta investigación concedemos importancia a la intuición de los hablantes respecto a la identidad de los fonemas en posición de neutralización. El motivo es precisamente funcional: estamos preocupados de la neutralización por sus consecuencias léxicas en un grupo de términos en los que se utilizan fonemas en eventual posición de neutralización para establecer nuevas oposiciones distintivas. Creemos que es necesario que los hablantes sean plenamente conscientes de las diferencias fonémicas en esta posición para poder atribuir valor distintivo a las mismas. 


\subsection{Neutralización de oposiciones multilaterales}

El concepto de neutralización desarrollado por Trubetzkoy considera que las oposiciones neutralizables deben ser bilaterales, es decir, aquellas en las cuales los rasgos comunes de los fonemas no sean compartidos por más fonemas en la lengua que aquellos cuya oposición se neutraliza. En una neutralización, los rasgos que definen la identidad del archifonema son sólo los rasgos pertinentes que comparten los fonemas neutralizados; una oposición neutralizable será siempre bilateral, debido a que si otro fonema en el sistema compartiera los rasgos pertinentes del archifonema, se confundiría con éste (Trubetzkoy, 1939, p. 70; Alarcos, 1965, p. 49).

Una parte de las neutralizaciones que ocurren en español funcionan de esta manera: las oposiciones de / pb/, / k-g/ y / r r / son bilaterales. Sin embargo, muchas de las neutralizaciones consignadas antes ocurren entre oposiciones multilaterales:

- en [op.'sion] / [ok.'sion] se neutralizan /p/ y / k/

- en [doh.'or] / [dok.'or] se neutralizan / k/ y /s/

- en [a.'moh.fe.ra] / [ah.'moh.fe.ra] se neutralizan /t/ y / s /

- en ['pi.sa] / ['pik.sa] se neutralizan /t / y / k/

- en [al.'kae] / [ar.'kar.ðe] / [ar.'kar.ðe] se neutralizan /1/, / / y /r/

- en ['fue.ra] / [xue.'ra] se neutralizan /f/ y / x/

- en ['buxea] y ['guxea] se neutralizan /b/ y /g/

- en [um.'pi.no] se neutralizan $/ \mathrm{n} / \mathrm{y} / \mathrm{m} /$

En todos estos casos, los rasgos comunes a las oposiciones neutralizadas son compartidos por otros fonemas: los rasgos comunes de $/ \mathrm{p} / \mathrm{y} / \mathrm{k} /$ también son compartidos por //; asimismo, los rasgos comunes de // y / $/$ son compartidos por $/ \mathrm{p} /{ }^{1}$; los rasgos comunes de /k/ y /s/, y los de // y /s/ son compartidos por /f/ y /x/; los rasgos

1 Podría pensarse que, si $/ \mathrm{p} / \mathrm{y} / \mathrm{k} /$ se neutralizan en la misma posición que $/ \mathrm{t} / \mathrm{y} / \mathrm{k} /$, entonces se trata de una neutralización de los tres fonemas. Sin embargo, en la práctica sucede que puede encontrarse la pronunciación [o.'sion] u [o.'sion], pero no *[o.'sion], aunque es perfectamente posible en este lugar de la sílaba, como lo comprueba su aparición en palabras como ritmo; y la pronunciación [a.'moh.fe.ra] y [a.'moh.fe.ra], pero no *[a.'moh.fe.ra], aunque también es posible en este lugar, en palabras como capturar. Esto nos habla de una neutralización entre pares de sonidos, y no entre tres sonidos. 
comunes de $/ \mathrm{f} / \mathrm{y} / \mathrm{x} /$ son compartidos por $/ \mathrm{s} / ;$ los rasgos comunes de /b/y /g/ son compartidos por /d/.

Los casos de $/ \mathrm{m}-\mathrm{n} / \mathrm{y}$ de $/ 1-\mathrm{r}-\mathrm{r} /$ son distintos a los anteriores. En el primero, el único sonido que comparte los rasgos comunes de $/ \mathrm{m} / \mathrm{y} / \mathrm{n} /$ es $/ \mathfrak{n} /$, que en español no aparece en coda silábica, por lo que también se considera parte del archifonema. El problema es que la distinción que establece Trubetzkoy entre oposiciones bilaterales y multilaterales es producto de la comparación entre pares de fonemas, por lo que las neutralizaciones también debieran producirse entre dos fonemas (1939, p. 60-61). Por el mismo motivo, el caso de /1, r, r / también resulta atípico.

Esta indicación de Trubetzkoy ha dado origen a una discusión sobre si las oposiciones multilaterales pueden conformar realmente una neutralización. Una panorámica general de la discusión de este problema puede hallarse en Rodríguez (1995)².

Algunos autores han agregado precisiones o excepciones a la descripción de Trubetzkoy para conservar la idea de que, en principio, sólo las oposiciones bilaterales se neutralizan, y al mismo tiempo, aceptar la neutralización de algunas oposiciones multilaterales (de / $1-r-r / y$ de $/ \mathrm{m}-\mathrm{n}-\mathrm{n} /$, particularmente, por ser las más extendidas del español). Por ejemplo, respecto del caso de las nasales, Alarcos afirma que "el carácter nasal de los fonemas $\mathrm{m} / \mathrm{n} / \mathrm{n}$ los coloca aparte de los fonemas orales y establece entre ellos oposiciones neutralizables" (1965, p. 181). Morales Pettorino (1976, p. 120), en su descripción de neutralizaciones en el español de Chile, explica la neutralización de nasales de este modo: al no encontrarse $/ \mathrm{n} /$ en posición codal, realmente se neutralizan sólo $/ \mathrm{m} / \mathrm{y} / \mathrm{n} /$. Sin explicitarlo, afirma que $/ \mathrm{m} / \mathrm{y} / \mathrm{n} /$ establecen aquí una oposición bilateral (dando por sentada la existencia de distintos sistemas fonológicos para distintos lugares de la sílaba).

Respecto del caso de / $1-r-r /$, Alarcos (1965) sugiere como explicación la ocurrencia de una doble neutralización: se neutralizarían primero oposiciones bilaterales, y luego los archifonemas resultantes. Así, según

2 Tal discusión sólo puede tener lugar desde una fonología no binarista, ya que en una descripción fonológica en base a rasgos binarios, todas las oposiciones son bilaterales, en ultima instancia; de este modo, Veiga (1993), que adhiere a una fonología binarista jakobsoniana, se refiere a neutralizaciones que abarcan numerosos fonemas en una sola unidad archifonémica. 
Alarcos, $/ \mathrm{l} / \mathrm{y} / \mathrm{K} / \mathrm{se}$ neutralizan en $/ \mathrm{L} / ; / \mathrm{r} / \mathrm{y} / \mathrm{s} /$ se neutralizan en $/ \mathrm{R} /$; finalmente, /L/ y / R/ también pueden neutralizarse a veces, en el habla vulgar o dialectal (si agrupamos todos estos fonemas por su carácter líquido, tendremos que la oposición / L - R/ es bilateral, ya que no hay otros sonidos en español que compartan este rasgo).

Martinet reelabora las doctrinas de Trubetzkoy a este respecto y modifica, entre otras cosas, este polémico punto: afirma que "La neutralización puede afectar a más de dos fonemas" (Martinet, 1984, p. 100), es decir, que no es necesario que la oposición neutralizada sea bilateral. Sus motivos provienen de una perspectiva completamente distinta de la neutralización y el archifonema. Trubetzkoy parte de la noción de fonema como unidad básica, y afirma que en algunas ocasiones pueden unirse dos fonemas en una misma función; es decir, compara fonemas y considera los rasgos como cualidades de los fonemas (como "base de comparación"). Posteriormente, como resultado de la verificación del hecho de la neutralización, llega a la noción del archifonema. Martinet toma otro camino: comienza con los rasgos, y a partir de la constatación del hecho de que en una posición los rasgos pertinentes son menos que en otra, establece la noción de archifonema. A diferencia de Trubetzkoy, no se refiere a un proceso de pérdida de distintividad, sino a uno en que se gana distintividad: según Martinet, el archifonema en algunas posiciones se escinde en dos fonemas. Esta solución parece una posición intermedia entre el multisistematismo y el concepto de neutralización y archifonema desarrollado por Trubetzkoy. De aquí surge que para Martinet no haya impedimento para que un archifonema reúna a más de dos fonemas. Sin embargo, conserva la idea de Trubetzkoy de que los fonemas que componen un archifonema deben tener en común un conjunto de rasgos que no compartan con otros fonemas del sistema; por esto, define al archifonema como el "conjunto de rasgos pertinentes comunes a dos o más fonemas que son los únicos que los presentan todos" (Martinet, 1984, p. 100).

Aunque estas explicaciones dan cuenta de la neutralización de $/ \mathrm{m}-\mathrm{n}-\mathrm{n} / \mathrm{y}$ de $/ \mathrm{l}-\mathrm{r}-\mathrm{r} /$, siguen dejando abierto el problema de las neutralizaciones de $/ \mathrm{p}-\mathrm{k} /, / \mathrm{k}-\mathrm{s} /, / \mathrm{s} /, / \mathrm{k} /, / \mathrm{f}-\mathrm{x} / \mathrm{y} / \mathrm{b}-\mathrm{g} /$, ya que en ellas participan fonemas que no son los únicos que presentan los rasgos pertinentes en común. El argumento de Trubetzkoy para plantear esta condición parece muy razonable y hace difícil explicar las 
neutralizaciones recién mencionadas: si un archifonema contiene los rasgos pertinentes comunes a la oposición fonológica que se neutraliza, entonces cualquier otro fonema que contenga esos rasgos debería formar parte del archifonema. No obstante, como hemos dicho, esto no parece ser exactamente lo que sucede en estos casos: aunque / s / compartiría los mismos rasgos pertinentes que el archifonema resultante de la neutralización de /f / y / x/, se puede encontrar ['fuxe.ra] y ['xuxe.ra], pero nunca *['sue.ra] (aunque [s] es perfectamente posible en ese contexto). Es decir, en realidad / s / no forma parte de la neutralización. Se objetará que en ['xue.ra] hay una motivación articulatoria que no existe en ['suxe. ra]; sin embargo, esa motivación tampoco existe en ['fune.ra] y eso no impide que se dé este caso. Además, en otros casos sucede algo parecido: se encuentra [ko.la.'sar] y [ko.la.'sar], pero no [ko.la.'sar]. Y aquí no hay motivación articulatoria alguna.

Son precisamente estas neutralizaciones las menos descritas en la bibliografía, y aparecen sólo en descripciones de algunos dialectos del español. Quizá el razonamiento que expone Obediente, a propósito de la neutralización de las oclusivas sordas y de / s - -y que lo lleva a concluir que allí no ocurre una verdadera neutralización-, es el mismo razonamiento que llevó a otros autores a no incluir neutralizaciones como éstas en sus inventarios. Estos espacios de confusión e imprecisión hacen necesario el estudio más detallado sobre el comportamiento de las neutralizaciones.

\section{Test percepción de neutralizaciones}

Esta investigación indaga sobre uno de los aspectos de la intuición de los hablantes sobre la identidad fonémica de los sonidos que aparecen en posición de neutralización. Consideraremos las neutralizaciones más comúnmente descritas para el castellano general y el dialecto chileno en particular: / p - b/, /d/, / k - g/, / r $-\mathrm{r} /, / \mathrm{m}-\mathrm{n}-\mathrm{n} /$ (que se neutralizan como norma general en el castellano), y / $-\mathrm{r}-\mathrm{s} / \mathrm{o} / \mathrm{l}-\mathrm{R} /, \mathrm{p}-\mathrm{k} /$, $/ \mathrm{k} /, / \mathrm{p}-\mathrm{s} /, / \mathrm{s} /, / \mathrm{k}-\mathrm{s} /, / \mathrm{b}-\mathrm{g} /, / \mathrm{f}-\mathrm{x} /$ (que se neutralizan en algunos dialectos).

Es necesaria todavía una investigación que muestre si realmente las oposiciones del segundo grupo se neutralizan en algunos dialectos, y en qué dialectos exactamente. Si -como afirma Veiga-, la distinción 
entre oclusivas en coda silábica es artificial, reflejo de la escritura, de modo que en todos los dialectos naturales del castellano ocurre una gran neutralización en esta posición, entonces los resultados de nuestra investigación ofrecerán información sobre la variación del grado de conciencia de los hablantes sobre la distintividad fonémica según el tipo de oposición que se neutralice. En caso contrario, si estas oposiciones no se neutralizan más que en dialectos minoritarios, cabría preguntarse si la conciencia de identidad fonémica es distinta en los grupos que neutralizan la oposición y en los que no lo hacen. Si la respuesta es positiva, esto podría ser un argumento a favor de la consideración de aspectos psicológicos en la descripción de la fonología de una lengua.

Esta investigación no puede responder a todas estas interrogantes, pero sí dar un paso necesario. El objetivo de este trabajo es determinar en qué medida los hablantes son conscientes del valor distintivo de los fonemas en distintas posiciones de neutralización que se han descrito para el castellano. Tener conciencia del valor distintivo de las unidades que participan en una neutralización es un aspecto vital para que puedan producirse los cambios que llevan al surgimiento de los proto-dobletes etimológicos que hemos mencionado al comienzo: es necesario que el hablante esté plenamente consciente de la diferencia fonológica entre dos formas para poder atribuir a éstas significados distintos. Dicho de otro modo, el diseño de esta investigación no tiene como objetivo describir el aspecto sistémico del fenómeno de la neutralización, sino un aspecto funcional: la potencialidad de las distintas neutralizaciones de ser percibidas como oposiciones fonológicas por los hablantes, y por tanto, la posibilidad de estos sonidos de distinguir pares mínimos. Sin embargo, los datos permiten hacer algunas consideraciones sobre la naturaleza y características de la neutralización que planteamos en los comentarios y que deberán ser respaldadas o refutadas por investigaciones posteriores que aborden las preguntas aquí sugeridas.

\subsection{Hipótesis}

A través de un test de percepción buscaremos comprobar las siguientes hipótesis:

1) Hay neutralizaciones en las cuales los hablantes están conscientes del valor distintivo de los fonemas que participan en ellas, y 
otras en las cuales los fonemas se confunden en una unidad indiferenciada.

2) Las oposiciones fonológicas que se neutralizan en una unidad segmental indiferenciada, que confunde los fonemas participantes, son oposiciones bilaterales. Las oposiciones multilaterales pueden neutralizarse, pero en estos casos no se produce realmente una confusión de los dos fonemas, sino que una suspensión del valor distintivo, consciente para el hablante.

3) A diferencia de lo que ocurre con las demás neutralizaciones multilaterales, los hablantes no son conscientes de las diferencias fonémicas de las nasales en posición de neutralización. Esta ausencia de consciencia está relacionada con el condicionamiento articulatorio de esta neutralización.

4) Las vibrantes en inicio de palabra son oposiciones bilaterales que no se perciben por parte de los hablantes.

\subsection{Diseño y aplicación de test}

En el test de percepción, se solicita a los informantes oír algunos enunciados grabados que contienen neutralizaciones y comparar esta grabación con la escritura ortográfica estándar del mismo enunciado (la realización fonética que se oye en la grabación no se corresponde con la escritura ortográfica de la palabra). Se solicitó a los informantes que escribieran sólo las palabras en las que escucharan algún sonido distinto del que aparece en la escritura, y que escribieran la palabra en que aparece ese sonido "tal como la escuchaban". El supuesto es que los hablantes se percatarían de la diferencia entre el audio y la escritura en las neutralizaciones en que tuvieran consciencia del valor distintivo de los fonemas participantes, mientras que no se percatarían en las neutralizaciones donde no estuvieran conscientes de la alternancia de más de un fonema, pues en esa posición estos fonemas constituyen para él una unidad indiferenciada.

Para poner a prueba la hipótesis 3, se generaron tres pares de enunciados que contienen neutralización de nasales: en el primer par, la nasal se asimila a la consonante siguiente en el punto de articulación; en segundo, la consonante nasal no se asimila a la consonante siguiente; en 
el tercero, la consonante aparece ante pausa. Para comprobar la hipótesis, los informantes debieran manifestar consciencia de la distinción entre $/ \mathrm{m} / \mathrm{y} / \mathrm{n} /$ en los casos en que no se asimila a la consonante siguiente, mientras que no debieran demostrarla cuando ocurra asimilación.

El test se implementó en una plataforma virtual, disponiendo para cada audio de un botón para reproducir la grabación a voluntad del informante, y bajo este botón un espacio para escribir las palabras en que se detectaran diferencias con el audio. Algunos datos se obtuvieron de forma presencial, dando a escuchar los estímulos por medio de un dispositivo reproductor de audio y proveyendo a los informantes de una pauta en papel donde anotar sus observaciones. Se agregaron algunos enunciados distractores, que no presentaban ninguna alternancia de fonemas, y otros que contenían alternancias que no corresponden a ninguna neutralización propia del español de Chile; estos enunciados, además de ocultar a los informantes el foco de la investigación, también sirvieron para identificar y descartar las respuestas de los informantes que no discriminaran los casos obvios de alteración de algún fonema o, por el contrario, que discriminaran y transcribieran incluso diferencias fonéticas propias del español de Chile.

Lo que en estos resultados se presenta como una sola muestra es en realidad la suma de los datos obtenidos en distintas muestras, compuestas del siguiente modo: 113 informantes para la mayoría de los casos, excepto la neutralización de / $1-\mathrm{r}-\mathrm{r} /$ (50 informantes), la de / $\mathrm{r}$ $-r$ / en inicio y final de palabra (24 informantes), y la neutralización de nasales en final de enunciado (52 informantes). Se restringió la muestra a personas que hablaran como primera lengua el español de Chile y que no se desempeñaran laboral o académicamente en ocupaciones relacionadas con el análisis del habla o del lenguaje.

\section{Resultados}

Los siguientes gráficos representan la proporción de informantes que son conscientes de las diferencias fonémicas en posición de neutralización (en negro), versus los que no son conscientes (en gris), en las distintas oposiciones que se neutralizan en español ${ }^{3}$.

3 Debido a que en español la neutralización de nasales ocurre con asimilación cuando hay una consonante posterior (los ejemplos sin asimilación evaluados en el test resultan enunciados poco probables), se utilizaron los datos de las neutralizaciones con asimilación para comparar la consciencia de los informantes en todas las neutralizaciones. 


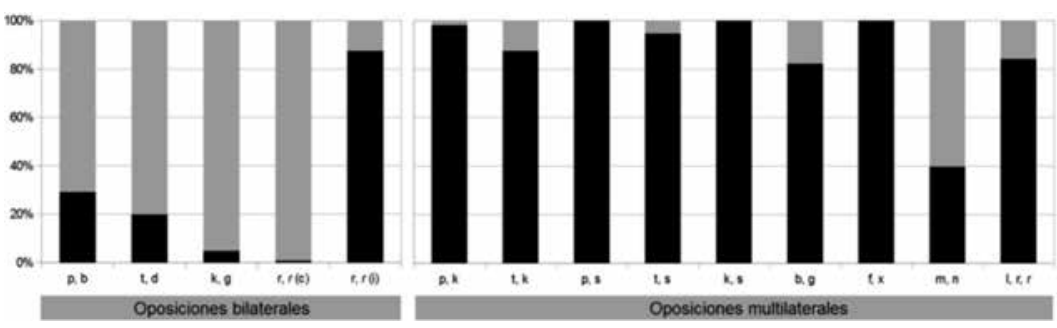

Gráfico 1. Consciencia de la identidad fonémica en la distintas neutralizaciones del castellano de Chile.

Se puede apreciar una diferencia notoria entre neutralizaciones en que se conserva la consciencia de identidad fonémica y otras en que no se conserva; el caso más cercano a la repartición equitativa de las dos opciones es en las nasales (en una proporción aproximada de 6:4). Exceptuando este caso, todos los demás están en una proporción de al menos 7:3. Estos datos confirman la hipótesis 1, demostrando que la consciencia de los hablantes respecto del valor fonémico de las unidades en posición de neutralización difiere significativamente en distintos casos de neutralización.

Además, se observa en el gráfico una tendencia a asociarse la conciencia de la identidad fonémica con las oposiciones multilaterales. La correlación entre el tipo de oposición que se neutraliza y la consciencia de los hablantes sobre la identidad fonémica es estadísticamente significativa al aplicar la prueba de chi cuadrado, con .01 de significatividad. Una razón que podría explicar la diferencia entre oposiciones bilaterales y multilaterales es que los rasgos que permiten distinguir un fonema de varios otros fonemas sean perceptualmente más prominentes. De este modo, si el punto de articulación en $/ \mathrm{p} /$ lo distingue de $/ \mathrm{k} /$, este rasgo será más prominente que el rasgo de sonoridad, puesto que éste sólo permite distinguirlo de /b/. Una oposición que se base en un rasgo menos prominente sería más débil que una oposición basada en un rasgo más prominente.

Ahora bien, debe tenerse en cuenta que estos datos podrían no estar interpretando lo que ocurre en general con la intuición del hablante respecto de los fonemas en neutralización, puesto que buena parte de estas neutralizaciones se describen como restringidas a algunas 
variedades sociales o situacionales, y esta investigación no se restringió a tales variedades. Los datos podrían estar describiendo solamente lo que un grupo mayoritario de hablantes percibe sobre usos dialectales minoritarios. En este caso, la debilidad de la oposición se relaciona con la extensión de la neutralización: una oposición más débil se neutralizará en todos los dialectos del castellano, mientras que una oposición menos débil lo hará sólo en algunos.

La neutralización de las nasales, tal como se previó en la hipótesis 3 , escapa de la norma de las multilaterales, pues solamente un 39,8\% de los informantes son conscientes de las diferencias fonémicas entre $/ \mathrm{m} / \mathrm{y} / \mathrm{n} /$ en posición de neutralización. En este punto, las nasales se comportan como las oposiciones bilaterales y, quizá por esto, distintos autores han buscado explicaciones para incluirlas en esta categoría. Otra excepción a esta tendencia es la neutralización de vibrantes en inicio de palabra. En este caso, una oposición bilateral resulta mayormente consciente para los hablantes en posición de neutralización (85,5\%).

\subsection{Excepciones: nasales y vibrantes}

Las nasales y las vibrantes en inicio de palabra constituyen la excepción a la tendencia antes descrita. Por esto, se recogieron otros datos sobre estos casos, aislando las variables de asimilación (en el caso de las nasales) y de posición en la palabra (en ambos casos). Los gráficos muestran la consciencia de los informantes sobre la identidad fonémica (en gris) para los siguientes casos: a) nasales en posición intermedia de palabra, asimiladas a la consonante siguiente (['al.ßue. fo.o. $\gamma$ ra.'fi.as], [sim. per.'mi.so]); b) nasales en posición intermedia de palabra, no asimiladas a la consonante siguiente ([kan.pe.'on], [im.'so.li.to]); c) nasales en posición final de palabra y de enunciado ([li.'mom], ['al.ßun]); d) vibrantes en posición intermedia de palabra; e) vibrantes en posición final de palabra y de enunciado; f) vibrantes en posición inicial de palabra. 

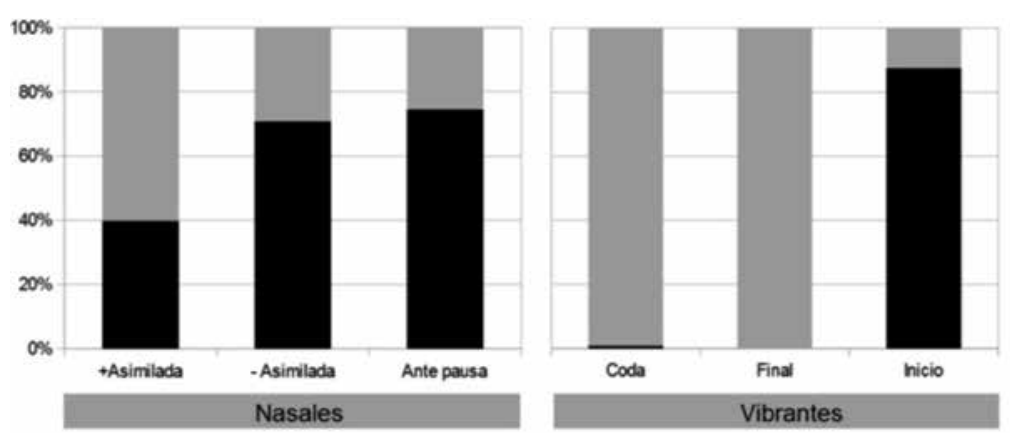

Gráfico 2. Consciencia de la identidad fonémica en nasales y vibrantes, considerando variables de asimilación y posición en la palabra.

Se puede observar que cuando la neutralización se realiza con la asimilación de la nasal al punto de articulación de la consonante siguiente, la consciencia de los hablantes sobre la diferencia de los fonemas es relativamente baja (39,8\%), mientras que en los dos contextos en que no se da asimilación, la mayor parte de los informantes están conscientes de la diferencia entre los fonemas (70,8\% ante consonante y $74,5 \%$ en final de enunciado). Es decir, cuando no ocurre asimilación, la neutralización de nasales es interpretada por los hablantes de la misma forma que las demás neutralizaciones multilaterales: la mayoría de ellos están conscientes de la diferencia entre los fonemas. A diferencia de lo que sucede con las nasales, en las vibrantes la neutralización al final de palabra se comporta del mismo modo que al interior de palabra: no es consciente para los hablantes. Estos datos parecen señalar que el factor crucial para que se produzca la diferencia de comportamiento de las nasales respecto de las demás oposiciones multilaterales es precisamente la asimilación.

Se podría objetar, sin embargo, que en la neutralización de / b - g/ y de / $\mathrm{f}-\mathrm{x}$ / también existe asimilación, y aún así los hablantes son conscientes de la diferencia de estos fonemas en posición de neutralización. No obstante, otra diferencia entre la neutralización de nasales y la de / b - g/ $\mathrm{y} / \mathrm{f}-\mathrm{x} /$ puede explicar el distinto grado de consciencia de los hablantes: $/ \mathrm{b}-\mathrm{g} / \mathrm{y} / \mathrm{f}-\mathrm{x} /$ se neutralizan en posición de ataque, mientras que la neutralización de nasales -al igual que la mayor parte de las restantes neutralizaciones- ocurre en distensión silábica, que es una posición más débil articulatoriamente (cfr., por ejemplo, Fernández-Sevilla, 1980). El comportamiento de las vibrantes nuevamente ofrece evidencia que 
apoya esta explicación: la neutralización de vibrantes a inicio de palabra es la única neutralización de oposición bilateral que es mayoritariamente consciente para los hablantes $(87,5 \%)$ y, coincidentemente, es la única que no aparece en posición de coda silábica.

De estos casos se desprende más claramente la existencia de varios factores que debilitan la oposición fonológica en el castellano. Ya habíamos identificado como factor de debilidad de oposición el hecho de que los fonemas conformen una oposición bilateral (es decir, se distingan por un rasgo menos prominente); ahora debemos agregar la asimilación fonética y la posición de coda silábica.

$\mathrm{Si}$ se analiza la combinatoria de estos factores en las distintas neutralizaciones que hemos mencionado y su relación con la consciencia de la identidad fonémica, se encontrará una constante. La mayoritaria consciencia de la identidad fonémica en la neutralización de $/ \mathrm{p}-\mathrm{k} /, \mathrm{k}$ $-\mathrm{s} / \mathrm{s} / \mathrm{s} / \mathrm{y} / \mathrm{l}-\mathrm{s}-\mathrm{r} /$ demuestra que la posición de coda por sí misma no es un factor de debilidad de oposición suficiente como para eliminar la percepción de las diferencias fonémicas. La mayoritaria consciencia sobre la identidad de $/ \mathrm{b}-\mathrm{g} / \mathrm{y} / \mathrm{f}-\mathrm{x} /$ en posición de neutralización demuestra que la asimilación tampoco es un factor determinante por sí mismo. La conjunción de dos condiciones de debilidad de la oposición, en cambio, puede evitar que los hablantes perciban las diferencia fonémicas. Así ocurre con / $\mathrm{p}-\mathrm{b} / \mathrm{,} / \mathrm{k}-\mathrm{g} / \mathrm{y} / \mathrm{r}-\mathrm{r} /$, cuya oposición fonológica deja de ser consciente para los hablantes cuando al factor de debilidad que implica conformar una oposición bilateral (y estar opuestas por un rasgo menos prominente), se agrega el factor de aparecer en distensión silábica. En el caso de las nasales (una oposición multilateral, es decir, más fuerte que una oposición bilateral), el hablante pierde consciencia de la identidad fonémica cuando aparece en distensión silábica y además se produce asimilación a la consonante posterior. En las vibrantes, como habíamos mencionado, los hablantes son conscientes de la diferencia fonémica sólo cuando aparece en inicio de palabra, dado que en esta posición el único factor de debilidad es ser una oposición bilateral.

Se puede apreciar, además, una relación entre la debilidad de la oposición y la generalidad de la neutralización. Las oposiciones que se ven afectadas por dos factores de debilidad, también son las que no tienen marcas dialectales que se neutralizan en todas las variedades del castellano (aunque lo contrario no es cierto siempre: la neutralización de 
$/ \mathrm{r}-\mathrm{r} /$ en inicio de palabra es generalizada en el castellano, pese a no ser afectada más que por un factor de debilidad).

\subsection{Relación de la consciencia de identidad fonémica con la neutralización}

Conociendo estos datos, estamos en condiciones de sugerir una explicación general para el proceso de neutralización y su comportamiento variable en lo que respecta a extensión a distintos dialectos y consciencia de los hablantes sobre la identidad fonológica de los fonemas involucrados. Por supuesto, debido a que los datos corresponden sólo a un dialecto del español, esta explicación es solamente una propuesta y necesita ser confirmada por el estudio de estos mismos factores en otras lenguas.

Como se ha mostrado previamente con ejemplos, la combinación de dos factores que debiliten la perceptibilidad atenúa la oposición, al grado de causar que los hablantes pierdan consciencia de la identidad de los fonemas que participan en ella. Estas oposiciones, no percibidas como tales por los hablantes, son inapropiadas para establecer distinciones léxicas, por lo cual la lengua carece de palabras que se opongan por estos fonemas en posición de debilidad. En conclusión, los fonemas no tienen posibilidad de conmutación en esa posición, lo cual es, para el criterio funcionalista, la evidencia de que la oposición se halla neutralizada. Las oposiciones debilitadas por dos factores se neutralizan generalizadamente en una lengua y en todos sus dialectos.

Ahora bien, oposiciones debilitadas por un solo factor no serán candidatas preferidas para establecer distinciones léxicas. Así, encontramos que la oposición de /f/ y / $/$ ante [u] no distinguen muchos pares mínimos (fuego y juego son quizá el único ejemplo en la lengua cotidiana); la oposición de /p/ y / k/ en distensión silábica distingue pares mínimos muy escasos, como aptitud y actitud, que son poco usuales en la lengua coloquial. La escasez de posibilidades de conmutación de los fonemas que conforman estas oposiciones en contexto fonético de debilidad -y el hecho de que los pocos casos de pares mínimos se encuentren en léxico especializado o cultismos- permite que estas oposiciones se neutralicen en la práctica de muchos dialectos o muchos registros del español. Esta neutralización, dependiendo de la aceptación de que goce como uso, podría ampliarse hasta abarcar todos los dialectos sociales de un dialecto geográfico, o incluso puede 
extenderse a la lengua completa, como sucede con la neutralización de $/ \mathrm{r}-\mathrm{r} / \mathrm{en}$ inicio de palabra.

De este modo, la extensión de la neutralización en los distintos dialectos de la lengua, y la consciencia de identidad fonémica, pueden explicarse conjuntamente si se considera la neutralización desde un punto de vista comunicativo-funcional, en lugar de describirla solamente como un fenómeno sistémico. Si la explicación que aquí planteamos es correcta, la posibilidad o imposibilidad de conmutación -criterio estrictamente sistémico para definir la neutralización- se relaciona estrechamente con factores comunicativos como la facilidad de percepción de la oposición. En este sentido, la fonología que otorga un papel importante a la intuición de los hablantes en la determinación de las unidades fonológicas no estaría alejada del concepto funcionalista del archifonema: los dos conceptos focalizan un momento diferente de este proceso, consideran como definitorias distintas evidencias.

\section{Conclusión}

Los resultados del test confirman la distinción entre dos grupos de neutralizaciones: uno en el cual los hablantes son conscientes de la identidad fonémica de las unidades que se neutralizan y otro en que no lo son. La consciencia de la identidad fonémica en posición de neutralización está estrechamente relacionada con la presencia de tres factores que debilitan la perceptibilidad de las oposiciones: a) conformar una oposición bilateral (por compartir la mayoría de los rasgos pertinentes y diferenciarse en uno poco prominente, ya que a cada término de la oposición le sirve para diferenciarse de un solo fonema en el sistema); b) ocupar una posición débil en la sílaba, como lo es la distensión; c) asimilarse a alguno de los segmentos circundantes. En todas las neutralizaciones que ocurren en castellano, se da al menos una de estas tres condiciones. Sólo cuando se presentan dos de ellas, los hablantes pierden consciencia de la identidad de los fonemas neutralizados.

La diferencia de comportamiento sociolingüístico de las neutralizaciones también se correlaciona con los tres factores recién descritos. Las neutralizaciones restringidas sólo a algunos dialectos del castellano corresponden a oposiciones que presentan sólo una condición de debilidad; las neutralizaciones extendidas a todos los dialectos, en 
cambio, casi todas presentan dos condiciones de debilidad (la excepción es la neutralización de $/ \mathrm{r}-\mathrm{r} /$ en inicio de palabra).

Dado que la asimilación es un factor poco frecuente, ocurre que la mayoría de las neutralizaciones generales al castellano resultan ser oposiciones bilaterales (la única excepción son las nasales). Si la poca frecuencia del factor asimilación se repite en otras lenguas, esto podría explicar la asociación estrecha que Trubetzkoy vio entre la neutralización y las oposiciones bilaterales. De hecho, las oposiciones bilaterales tienen muchas más probabilidades de neutralizarse.

Se puede sostener que la pérdida de consciencia de la identidad fonémica es producto de la conjunción de distintos factores de debilidad de percepción de la oposición. La neutralización no se corresponde siempre con la pérdida de perceptibilidad de las oposiciones fonémicas, pero en la mayor parte de los casos puede explicarse la imposibilidad de conmutación de algunos fonemas en ciertas posiciones por la debilidad de la oposición. De este modo, si bien es cierto, la neutralización se define por criterios sistémicos, también es cierto que la realidad sistémica que la define (la posibilidad de conmutación) puede estar determinada por factores comunicativos y pragmáticos, como lo son la consciencia de la oposición y la presencia de factores fonéticos que debilitan la perceptibilidad de la oposición.

Esta concepción de la neutralización no corresponde completamente con la definición más habitual dentro del funcionalismo de la escuela de Praga. Esta escuela suele ver en la neutralización un fenómeno principalmente sistémico, paradigmático, un asunto de relación entre sonidos (en consonancia con su definición del fonema, despojada de alusiones a la intuición del hablante); sin embargo, la consideración de factores comunicativos -que incluyen la consciencia del hablante sobre la diferencia fonémica y las particularidades fonéticas que diluyen las diferencias entre los fonemas en oposición- ofrece una explicación razonable de la naturaleza y causas del fenómeno, y no es contraria a los principios declarados del funcionalismo de estudiar la lengua con especial atención en su función comunicativa.

Considerando que algunas de las neutralizaciones estudiadas ocurren sólo en algunos dialectos, y que cada dialecto es un sistema distinto, en estricto rigor no se puede extrapolar a todo sistema la afirmación 
de que la conjunción de dos factores de debilidad ocasiona la pérdida de consciencia de la oposición. Sería necesario e interesante medir esta variable en hablantes que efectivamente presenten las neutralizaciones restringidas dialectalmente.

Finalmente, y respecto al problema que motivó la investigación -es decir, la presencia de un valor distintivo potencial en algunas neutralizaciones fonémicas-, se concluye que las neutralizaciones en que sólo se da un factor de debilidad en general, servirían para establecer algunas oposiciones léxicas, pues el hablante sigue siendo consciente de la identidad fonémica (de hecho, algunas veces lo hacen en la lengua, mayormente en cultismos). Pero la consciencia de la identidad no es el único factor relevante. Si una oposición, pese a ser consciente para los hablantes -y por lo tanto, no estar obligada a neutralizarse-, de todas maneras se neutraliza generalizadamente, tampoco servirá como potencial unidad distintiva. Tal es el caso de las vibrantes a inicio de palabra en español. Sólo tienen valor distintivo potencial las oposiciones que se neutralizan ocasionalmente: $/ 1-R /, / \mathrm{p}-\mathrm{k} /, / \mathrm{k} /, / \mathrm{p}-\mathrm{s} /, / \mathrm{s} /$, $/ \mathrm{k}-\mathrm{s} /, / \mathrm{b}-\mathrm{g} /, / \mathrm{f}-\mathrm{x} /$. Tales neutralizaciones, además, suelen funcionar como estereotipos de grupo o presentar una distribución situacional restringida, lo cual permite que se den casos en que el valor distintivo de los fonemas se utiliza para conformar nuevas oposiciones léxicas en la lengua coloquial (doctor / dostor, último / úrtimo, buena / güena, fuerte / juerte y humilde / humirde), aprovechando las diferencias en la distribución situacional de las acepciones de las palabras. 


\section{Bibliografía}

Alarcos, Emilio. (1965). Fonología española (4ta edición). Madrid: Gredos. Anderson, James. (1991). Doublets, cultismos and their relation in castilian spanish, Orbis, 35, 166-170.

Burquest, Donald. [2006] (2009). Análisis fonológico. Un enfoque funcional. Traducción de Giuliana López Torres. Dallas: SIL International.

Cepeda, Gladys. (1994). Las consonantes del español de Valdivia (Chile). Los procesos de reforzamiento y debilitamiento fonológicos. Estudios Filológicos, 29, 39-61.

Cifuentes, Edgardo. (2015). Algunos dobletes etimológicos en formación en el español de Chile: opinión de los hablantes respecto a sus significados. Manuscrito en evaluación para su publicación.

Fernández-Sevilla, Julio. (1980). Los fonemas implosivos en español. Thesaurus, XXXV(3), 456-505.

Granados, Héctor. (2001). La neutralización de /r/ y /1/ en el dialecto oriental del español de Venezuela: más allá del sistema de la lengua. Lingua Americana, 8, 28-44.

Gutiérrez, Salvador. (1983). La Fonología. En Francisco Abad y A. García Berrio (Coords.). Introducción a la lingüística (pp. 75112). Madrid: Alhambra.

Martinet, Andre. (1984). Elementos de lingüística general. Madrid: Gredos.

Morales Pettorino, Félix. (1976). La neutralización consonántica en el español de Chile. Estudios Filológicos, 11, 113-128.

Obediente, Enrique. (1998). Fonética y fonología. Mérida: Universidad de Los Andes.

Pike, Keneth. [1947] (1961). Phonemics a technique for reducing languages to writing. Ann Arbor: The University of Michign Press.

Quilis, Antonio. 1999. Tratado de fonología y fonética españolas. Madrid: Gredos. 
Rodríguez, Bonifacio. (1990). La neutralización en fonología: neutralización y archifonema (I). Contextos, VIII(1516), 111-127.

- (1995). La neutralización en fonología: neutralización y archifonema (II). Contextos, XIII(25- 26), 41-56.

Sapir, Edward. (1972). La realidad psicológica de los fonemas. En N. Trubetzkoy, E. Sapir y otros. Fonología y morfología (pp. 31-46). Buenos Aires: Paidos.

Stockwell, Robert y Donald Bowen. (1965). The sounds of English and Spanish. Chicago: The University of Chicago Press.

Trubetzkoy, Nikolai. 1987 [1939]. Principios de fonología. Madrid: Cincel.

Veiga, Alexandre. (1984). Don unidades del sistema fonológico del español: El fonema africado y el archifonema interrupto. Verba, 11, 157-179.

. (1993). En torno a los fenómenos fonológicos neutralización y distribución defectiva. Verba, 20, 113-140.

. (1995). Los fonemas de realización nasal en español. Moenia, 1, 345-366.

Wright, Roger. ([1982] 1989). Latín tardío y romance temprano (en España y la Francia Carolingia). Madrid: Gredos. 